\title{
BLOWING UP THE POWER SET OF THE LEAST MEASURABLE
}

\author{
ARTHUR W. APTER AND JAMES CUMMINGS
}

\begin{abstract}
We prove some results related to the problem of blowing up the power set of the least measurable cardinal. Our forcing results improve those of [1] by using the optimal hypothesis.
\end{abstract}

\section{INTRODUCTION}

In his paper "Forcing the least measurable to violate GCH" [1] the first author proved the consistency of the following situation: $\kappa$ is the least measurable cardinal, $2^{\kappa}=\kappa^{+}$, and $\kappa$ remains the least measurable cardinal after forcing with the Cohen poset for adding $\kappa^{++}$subsets of $\kappa$. The construction of [1] starts with a model in which GCH holds and $\kappa$ is $\kappa^{+}$-supercompact, and proceeds by an iteration of Prikry forcing and Cohen forcing in the style of Gitik $[4,6]$.

It is known by work of Gitik [5], Mitchell [11] and Woodin (unpublished) that the exact strength of the failure of $\mathrm{GCH}$ at a measurable cardinal is given by the existence of a cardinal $\lambda$ of Mitchell order $\lambda^{++}$. It is clear that the result mentioned in the first paragraph can not be done from a weaker hypothesis than this; our main result is that this hypothesis suffices. Along the way we will prove some other results related to the problem of violating $\mathrm{GCH}$ at a measurable cardinal.

Silver gave the first consistency proof for the failure of the $\mathrm{GCH}$ at a measurable cardinal; his argument involved starting with a very large (supercompact) cardinal $\kappa$ and then doing a Reverse Easton iteration in which $\alpha^{++}$Cohen subsets are added to each inaccessible $\alpha \leq \kappa$. In

2000 Mathematics Subject Classification. Primary 03E35, 03E55; Secondary $03 \mathrm{E} 05$.

Key words and phrases. Measurable cardinal, GCH, Reverse Easton forcing, inner model.

The first author's research was partially supported by PSC-CUNY Grants 669408, 667379, and 61449-00-30, and by the Volkswagen-Stiftung (RiP-program at Oberwolfach).

The second author's research was partially supported by NSF grants DMS9703945 and DMS-0070549, and by the Volkswagen-Stiftung (RiP-program at Oberwolfach). 
what follows we refer to Silver's forcing construction as the standard iteration, and say that the standard iteration succeeds at a measurable cardinal $\kappa$ if it preserves the measurability of $\kappa$.

Woodin showed that it is possible to violate GCH starting with $\kappa$ which is $(\kappa+2)$-strong, but his construction (whose ideas we will use in this paper) is more complex than the standard iteration. Gitik's construction from the optimal hypothesis $o(\lambda)=\lambda^{++}[5]$ is even more complex, involving in addition a preliminary Prikry-style forcing.

As part of the proof of the main result, we will show that it is possible to start from a canonical inner model for $o(\lambda)=\lambda^{++}$and to build a model over which the standard iteration succeeds. We also show that no forcing resembling the standard iteration can succeed over this canonical inner model itself. Finally we show that a technical hypothesis on a measurable cardinal $\kappa$ introduced by Woodin is consistent with $\kappa$ being the least measurable cardinal.

Note on terminology: Our conventions are fairly standard. In particular in a forcing poset $p \leq q$ means that $p$ is stronger than $q$. A $\kappa$-closed poset is one in which every sequence of length less than $\kappa$ has a lower bound. A $\kappa$-Knaster poset is one in which every $\kappa$-sequence of conditions has a subsequence of length $\kappa$ consisting of pairwise compatible elements. If $\mathbb{P}_{\alpha}$ is an iteration of length $\alpha$ and $\beta<\alpha$ then $\mathbb{P}_{\beta}$ is the subiteration of $\mathbb{P}_{\alpha}$ through stage $\beta$; if $G_{\beta}$ is $\mathbb{P}_{\beta}$-generic then $\mathbb{P}_{\beta, \alpha}$ is the standard factor iteration as computed in $V\left[G_{\beta}\right]$.

\section{An inNer MODEL ARgument}

As we mentioned in the introduction, Gitik [5] has shown how to start with a canonical inner model for the hypothesis $o(\kappa)=\kappa^{++}$and produce a model in which $\kappa$ is measurable and $2^{\kappa}=\kappa^{++}$. The first step in Gitik's construction is an Easton support iteration of Prikrystyle forcing in which many measurable cardinals are made singular and extension is defined in a special way.

It is natural to ask whether this is really necessary, or whether instead it may be possible to get the desired result by the classical technique of iterating highly closed forcing with Easton support. We show here that this is not the case when forcing over a certain canonical inner model for the hypothesis $o(\kappa)=\kappa^{++}$.

We will use a standard fact from inner model theory. It is shown in Mitchell's papers on inner models constructed from sequences of measures $[11,9,10]$ that if there is an inner model in which $o(\lambda)=\lambda^{++}$ for some cardinal $\lambda$, then we may construct an inner model $V$ with the following pleasant properties. 
(1) $V$ models GCH.

(2) There is a sequence $U(\alpha, \beta)$ such that

(a) $U(\alpha, \beta)$ is defined for every $\beta<o^{U}(\alpha)$, for some ordinalvalued function $o^{U}$.

(b) $U(\alpha, \beta)$ is a normal measure on $\alpha$.

(c) $o^{U}(\kappa)=\kappa^{++}$and $\kappa$ is the largest point at which $o^{U}$ is non-zero.

(3) If $V[G]$ is any set-generic extension of $V, W$ is any measure on $\kappa$ and $i: V[G] \longrightarrow N$ is the corresponding ultrapower map then $i \uparrow V$ arises from some iteration of $V$ with the following properties:

(a) The critical points in the iteration are increasing.

(b) Direct limits are taken at limit stages.

(c) If $j_{0 \gamma}: V \longrightarrow M_{\gamma}$ is the map at stage $\gamma$ in the iteration then $M_{\gamma+1}$ is the ultrapower by some measure $j_{0 \gamma}(U)(\alpha, \beta)$ and $j_{\gamma \gamma+1}$ is the corresponding ultrapower map.

We can now state the main result of this section.

Theorem 2.1. Let $V$ have the properties listed above. Then there is no poset $\mathbb{P} \in V$ such that

(1) Forcing over $V$ with $\mathbb{P}$ adds no $\omega$-sequences of ordinals.

(2) In $V^{\mathbb{P}}$ the cardinal $\kappa$ is measurable and $2^{\kappa}=\kappa^{++}$.

Proof. We proceed by contradiction. Let $G$ be $\mathbb{P}$-generic, and suppose that in $V[G]$ the cardinal $\kappa$ is measurable and $2^{\kappa}>\kappa^{+}$. Let $W$ be any normal measure on $\kappa$ and let $i: V[G] \longrightarrow N$ be the associated ultrapower map. By standard facts about measurability we see that $i(\kappa)>\left(2^{\kappa}\right)_{V[G]}$. By hypothesis $\left(2^{\kappa}\right)_{V[G]} \geq \kappa_{V[G]}^{++}$, and clearly $\kappa_{V[G]}^{++} \geq$ $\kappa^{++}$. We conclude that $i(\kappa)>\kappa^{++}$.

Now let $H=i(G), j=i \uparrow V$ and $M=\bigcup_{\alpha} i\left(V_{\alpha}\right)$. It is routine to check that $M$ is a transitive model of set theory, $H$ is $j(\mathbb{P})$-generic over $M, N=M[H]$ and $j: V \longrightarrow M$ is elementary. By standard facts about measurability $V[G] \models{ }^{\kappa} N \subseteq N$.

Let $j: V \longrightarrow M$ be generated by an iteration as above of length $\alpha$. Since the critical points are increasing, the first critical point in the iteration must be $\kappa$.

Claim. $\alpha$ is finite.

Proof. Suppose not and let $x=\left\langle\kappa_{i}: i<\omega\right\rangle$ be the sequence of the first $\omega$ many critical points. Now $x \in V[G]$, and so by the closure of $N$ we have $x \in N$. Since $N=M[H]$ and by elementarity the poset $j(\mathbb{P})$ adds no $\omega$-sequences of ordinals, $x \in M$. Since the critical points in the 
iteration are increasing, $x$ is in the model $M_{\omega}$ which appears at stage $\omega$ in the iteration.

Since $M_{\omega}$ is constructed as a direct limit, $x=j_{n \omega}(y)$ for some $n<\omega$ and $y \in M_{n}$. In particular the $n$-th point of $x$ must be the image of the $n$-th point of $y$. This is impossible because that $n$-th point is $\kappa_{n}$, which can not be in the range of $j_{n \omega} \operatorname{since} \operatorname{crit}\left(j_{n \omega}\right)=\kappa_{n}$.

Let $\alpha=n$ for some finite $n$. Since $\kappa$ is the largest $\lambda$ such that $o^{U}(\lambda) \neq 0$, it is easy to see that $\kappa_{i} \leq j_{0 i}(\kappa)$ for all $i<n$. Since $M_{n}=M$ is constructed by a finite iteration using normal measures, we also see that

$$
M_{n}=\left\{j(F)\left(\kappa_{0}, \ldots, \kappa_{n-1}\right): F \in V, \operatorname{dom}(F)=[\kappa]^{n}\right\} .
$$

Since GCH holds in $V$, there are only $\kappa^{+}$functions from $[\kappa]^{n}$ to $\kappa$. It follows that $j(\kappa)<\kappa^{++}$, which is absurd because $j(\kappa)=i(\kappa)$ and $i(\kappa)>\kappa^{++}$. This contradiction finishes the proof.

\section{Arguments of Woodin And Levinski}

In this section we outline arguments of Woodin and Levinski, which will be needed for the forcing construction of Section 4 .

3.1. Woodin's argument. Woodin invented a forcing technique for starting with a certain fairly weak embedding hypothesis on $\kappa$, and producing a model in which $\kappa$ is measurable and $2^{\kappa}=\kappa^{++}$. For more details we refer the reader to Gitik's paper [5] which shows that Woodin's hypothesis is consistent relative to $o(\kappa)=\kappa^{++}$, and then explains in detail Woodin's methods for getting the failure of GCH at a measurable and the failure of SCH at $\aleph_{\omega}$ from this hypothesis. In particular, [5, pages 227-229] contains the argument we are about to outline below.

The hypothesis we make on $\kappa$ is that GCH holds and there is an embedding $j: V \longrightarrow M$ such that $\operatorname{crit}(j)=\kappa,{ }^{\kappa} M \subseteq M$ and $\kappa_{M}^{++}=$ $\kappa^{++}$. We note that the existence of such an embedding is immediate if $\kappa$ is $(\kappa+2)$-strong.

We will begin by doing a Reverse Easton iteration $\mathbb{P}$ of length $\kappa$, in which $\alpha^{++}$subsets are added to each $\alpha<\kappa$. Let $G$ be generic for $\mathbb{P}$. Now let $\mathbb{Q}=A d d\left(\kappa, \kappa^{++}\right)_{V[G]}$ and let $g$ be $\mathbb{Q}$-generic over $V[G]$.

We will factor $j$ through the ultrapower by the normal measure $U=$ $\{X: \kappa \in j(X)\}$. Let $i: V \longrightarrow M_{0}=U l t(V, U)$ be the canonical ultrapower map, and let $k: M_{0} \longrightarrow M$ be the map given by $k$ : $[F]_{U} \longmapsto j(F)(\kappa)$. It is easy to see that $k$ is elementary, $k \circ i=j$ and $\operatorname{crit}(k)=\kappa_{M_{0}}^{++}$. We let $\lambda=\kappa_{M_{0}}^{++}$and note that by GCH and elementarity $\lambda<i(\kappa)<\kappa^{++}$. 
Now let $g_{0}$ be the $A d d(\kappa, \lambda)$-generic obtained by restricting $g$, and observe that $2^{\kappa}=\kappa^{+}$in $V\left[G * g_{0}\right]$. Let $\mathbb{R}=\operatorname{Add}\left(\kappa^{+}, \kappa^{++}\right)_{V\left[G * g_{0}\right]}$ and let $h$ be $\mathbb{R}$-generic over $V[G * g]$. Our final model will be $V[G * g * h]$. We note for the record that $V[G * g]$ is an extension of $V\left[G * g_{0}\right]$ by $\kappa^{+}$-c..c forcing, and so by Easton's lemma forcing with $\mathbb{R}$ over $V[G * g]$ adds no $\kappa$-sequences of ordinals.

The iteration $i(\mathbb{P})$ in $M_{0}$ may be factored as $\mathbb{P} * \dot{\mathbb{Q}}_{0} * \dot{\mathbb{S}}_{0}$ where $\mathbb{Q}_{0}=$

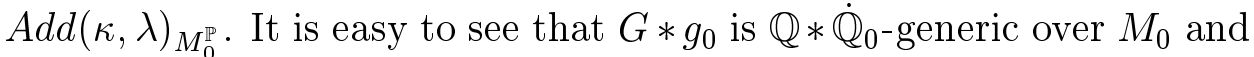
that $V\left[G * g_{0}\right] \models{ }^{\kappa} M_{0}\left[G * g_{0}\right] \subseteq M_{0}\left[G * g_{0}\right]$ We may use the standard method to build $H_{0} \in V\left[G * g_{0}\right]$ which is $\mathbb{S}_{0}$-generic over $M_{0}\left[G * g_{0}\right]$.

It is routine to lift $k: M_{0} \longrightarrow M$ to a new map $k^{+}: M_{0}\left[G * g_{0}\right] \longrightarrow$ $M[G * g]$, and to see that

$$
M[G * g]=\left\{k^{+}(F)(a): F \in M_{0}\left[G * g_{0}\right], a \in\left[\kappa^{++}\right]^{<\omega}, \operatorname{dom}(F)=[\lambda]^{<\omega}\right\} .
$$

Since $\mathbb{S}_{0}$ is $\lambda^{+}$-closed in $M\left[G * g_{0}\right]$ we may transfer $H_{0}$ along $k^{+}$. The result is a filter $H$ which is generic over $M[G * g]$ for $\mathbb{S}$, where $\dot{\mathbb{S}}$ is the last term in the factorisation $j(\mathbb{P})=\mathbb{P} * \dot{\mathbb{Q}} * \dot{\mathbb{S}}$. We may now lift $k^{+}$to $k^{++}: M_{0}\left[G * g_{0} * H_{0}\right] \longrightarrow M[G * g * H]$, and may also lift $j$ to $j^{+}: V[G] \longrightarrow M[G * g * H]$.

Since $H_{0} \in V\left[G * g_{0}\right]$, we see that $V\left[G * g_{0}\right] \models{ }^{\kappa} M_{0}\left[G * g_{0} * H_{0}\right] \subseteq$ $M_{0}\left[G * g_{0} * H_{0}\right]$. We may lift $i$ to get $i^{+}: V[G] \longrightarrow M_{0}\left[G * g_{0} * H_{0}\right]$, and may use the closure of $M_{0}\left[G * g_{0} * H_{0}\right]$ to argue that in $V\left[G * g_{0}\right]$ the forcing $i^{+}(\mathbb{Q})$ is equivalent to $\mathbb{R}$. We may therefore find $h^{*} \in$ $V[G * g * h]$ which is $V\left[G * g_{0}\right]$-generic for $i^{+}(\mathbb{Q})$. Note that a fortiori $h^{*}$ is $M_{0}\left[G * g_{0} * H_{0}\right]$-generic for $i^{+}(\mathbb{Q})$.

We now transfer $h^{*}$ along $k^{++}$to get $h^{* *}$ which is $M[G * g * H]$-generic for $j^{+}(\mathbb{Q})$. Unfortunately $h^{* *}$ is probably not compatible with $g$ and $j^{+}$, but we may alter $h^{* *}$ to obtain $h^{* * *}$ which is still $M[G * g * H]$-generic for $j^{+}(\mathbb{Q})$ and is also such that $j^{+} " g \subseteq h^{* * *}$.

We may now lift $j^{+}$to $j^{++}: V[G * g] \longrightarrow M\left[G * g * H * h^{* * *}\right]$. We are still not quite done, but since $\mathbb{R}$ is sufficiently distributive we may transfer $h$ along $j^{++}$to obtain $h^{\dagger}$ which is $j^{++}(\mathbb{R})$-generic over $M\left[G * g * H * h^{* * *}\right]$. We now lift to get $j^{+++}: V[G * g * h] \longrightarrow$ $M\left[G * g * H * h^{* * *} * h^{\dagger}\right]$. This witnesses that $\kappa$ is measurable in $V[G * g * h]$, and we are done.

3.2. Levinski's argument. Levinski [8] showed that the situation in which $\kappa$ is measurable and GCH holds at $\kappa$ while failing unboundedly often below is consistent relative merely to the existence of a measurable cardinal. We outline a version of the argument here. 
Suppose that $\kappa$ is measurable and GCH holds. Let $\mathbb{P}_{\kappa}$ be a Reverse Easton iteration in which $\alpha^{++}$Cohen subsets are added to each inac-

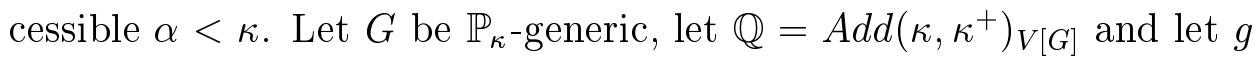
be $\mathbb{Q}$-generic over $V[G]$.

Let $U$ be a normal measure on $\kappa$ and let $i: V \longrightarrow M=U l t(V, U)$ be the associated ultrapower map. Let $\lambda=\kappa_{M}^{++}$and observe that $\kappa^{+}<\lambda<\kappa^{++}$. We may therefore find $g^{*} \in V[G * g]$ which is $\operatorname{Add}(\kappa, \lambda)$ generic over $V[G]$ and is such that $V[G * g]=V\left[G * g^{*}\right]$.

Arguing as in the last subsection we may build $H \in V[G * g]$ which is generic over $M\left[G * g^{*}\right]$ for $i\left(\mathbb{P}_{\kappa}\right)_{\kappa+1, i(\kappa)}$, and may then extend to get a map $i^{+}: V[G] \longrightarrow M\left[G * g^{*} * H\right]$.

Since $\operatorname{Add}\left(\kappa, \kappa^{+}\right)$has $\kappa^{+}$maximal antichains and $\left|j\left(\kappa^{+}\right)\right|=\kappa^{+}$, we may also build $h \in V[G * g]$ which is $i^{+}(\mathbb{Q})$-generic over $M\left[G * g^{*} * H\right]$. The construction may be done in a way such that $i^{+}$" $g \subseteq h$.

We may now lift again to get $i^{++}: V[G * g] \longrightarrow M\left[G * g^{*} * H * h\right]$. This shows that $\kappa$ is measurable in $V[G * g]$.

\section{A FORCING ARGUMENT}

In this section we show how to begin with a canonical model with $o(\kappa)=\kappa^{++}$of the sort discussed in Section 2 and to create by forcing a model in which $2^{\kappa}=\kappa^{+}, \kappa$ is the least measurable and $\kappa$ remains the least measurable after adding $\kappa^{++}$Cohen subsets of $\kappa$. The forcing construction proceeds in a number of stages, some of which are quite standard and will not be described in any detail.

Stage One: We do the iterated Prikry-style forcing described in Gitik's paper [5]. After this forcing we have a universe in which $\mathrm{GCH}$ holds and there is an embedding $j: V \longrightarrow M$ such that $\operatorname{crit}(j)=\kappa$, ${ }^{\kappa} M \subseteq \kappa$ and $j(\kappa)>\kappa^{++}$.

We claim that without loss of generality we may assume that $\kappa$ is not measurable in $M$. If $\kappa$ is measurable in $M$ then there is $U \in M$ which is a measure of Mitchell order zero, that is to say $\kappa$ is not measurable in $\operatorname{Ult}(M, U)$. We may now replace $M$ by $U l t(M, U)$ and $j$ by the composite embedding $i_{U}^{M} \circ j$, where $i_{U}^{M}$ is the canonical ultrapower embedding from $M$ to $\operatorname{Ult}(M, U)$.

Now let $E$ be the $\left(\kappa, \kappa^{++}+1\right)$-extender approximating $j$. Let $j_{E}$ : $V \longrightarrow U l t(V, E)$ be the ultrapower of $V$ by $E$ and $k: U l t(V, E) \longrightarrow M$ the standard map such that $k \circ j_{E}=j$. We know that $j_{E}(\kappa)>\kappa^{++}$ and also $\operatorname{crit}(k)>\kappa^{++}$, so that in particular $k(\kappa)=\kappa$ and $\kappa$ is not measurable in $\operatorname{Ult}(V, E)$. Standard arguments also show that $U l t(V, E)$ is closed under $\kappa$-sequences. 
Replacing $j$ by $j_{E}$, we may assume that in addition to the properties of $j$ listed above, $j$ arises as the ultrapower by a $\left(\kappa, \kappa^{++}+1\right)$-extender.

Stage Two: We force with Woodin's "fast function" forcing. The conditions are partial functions $p$ from $\kappa$ to $\kappa$ such that

- The domain of $p$ consists of inaccessible cardinals $\gamma<\kappa$ which are closed under $p$. That is to say if $\alpha$ and $\beta$ are in $\operatorname{dom}(p)$ and $\alpha<\beta$ then $p(\alpha)<\beta$.

- For every $\beta \in \operatorname{dom}(p)$, the cardinality of $\operatorname{dom}(p) \cap \beta$ is less than $\beta$.

The ordering is inclusion. We refer the reader to Hamkins' paper [7] for a detailed discussion of this forcing. We note that by a standard $\Delta$-system argument, since $\kappa$ is Mahlo the fast function forcing has the $\kappa$-Knaster property.

Let $\mathbb{P}$ be the fast function forcing, let $G$ be $\mathbb{P}$-generic, and let $f$ : $\kappa \longrightarrow \kappa$ be the fast function added by $G$. Arguing as in Theorem 1.6 of [7] we may build $H \subseteq j(\mathbb{P})$ such that

(1) $H$ is $j(\mathbb{P})$-generic over $M$.

(2) $j$ " $G \subseteq H$.

(3) The condition $\left\{\left(\kappa, \kappa^{++}\right)\right\}$is in $H$.

The arguments given in [7] show that $G$ is $\mathbb{P}$-generic over $M$, and the models $M[G]$ and $M[H]$ agree up to at least the rank of the first $M$ inaccessible greater than $\kappa$. Also, by Theorem 1.3 of [7], GCH holds in $V[G]$.

We may lift $j$ to get a new map $j^{+}: V[G] \longrightarrow M[H]$ such that $j^{+}(f)(\kappa)=\kappa^{++}$. It is a standard fact that $j^{+}$arises as the ultrapower by a $\left(\kappa, \kappa^{++}+1\right)$ extender, and since $\kappa^{++}=j^{+}(f)(\kappa)$ we see that $j^{+}$ is actually the ultrapower by a $\left(\kappa, \kappa^{++}\right)$-extender.

We claim that $\kappa$ is not measurable in $M[H]$. It follows by the factorisation argument of Lemma 1.1 in [7] that it is enough to show that $\kappa$ is not measurable in $M[G]$. This is immediate because $\mathbb{P}$ is $\kappa$-Knaster in $M$, and by a well-known argument (see for example [2]) a $\kappa$-Knaster forcing can not create measurability at $\kappa$.

We also claim that $M[H]$ is closed under $\kappa$-sequences inside $V[G]$. Since these are models of ZFC it is enough to check that every $\kappa$ sequence of ordinals from $V[G]$ is in $M[H]$. This is immediate because $V \models{ }^{\kappa} M \subseteq M$, and since $G$ is generic for $\kappa$-c.c forcing it follows that $V[G] \models{ }^{\kappa} O N \subseteq M[G]$.

To simplify notation we relabel so that $V[G]$ becomes $V, j^{+}$becomes $j$ and $M[H]$ becomes $M$. We are now in the following situation: $\mathrm{GCH}$ holds and there is $j: V \longrightarrow M$ such that $\operatorname{crit}(j)=\kappa,{ }^{\kappa} M \subseteq M, \kappa$ is 
not measurable in $M, j$ is the ultrapower by a $\left(\kappa, \kappa^{++}\right)$-extender and there is $f: \kappa \longrightarrow \kappa$ such that $j(f)(\kappa)=\kappa^{++}$.

Stage Three: We now do a Reverse Easton iteration $\mathbb{P}_{\kappa+1}$, in which we force at $\alpha \in \operatorname{dom}(f)$ with the Levy collapse $\operatorname{Coll}\left(\alpha^{+},<f(\alpha)\right)$ and then force at $\kappa$ with $\operatorname{Coll}\left(\kappa^{+},<\kappa^{++}\right)$. We recall from the previous stage that if $\alpha \in \operatorname{dom}(f)$ then $\alpha$ is inaccessible and $f$ " $\alpha \subseteq \alpha$.

Let $G$ be $\mathbb{P}_{\kappa}$-generic over $V$, and let $g$ be generic over $V[G]$ for $\operatorname{Coll}\left(\kappa^{+},<\kappa^{++}\right)$. We note that $g$ does not collapse cardinals, since $V[G]$ models $\mathrm{GCH}$ and $\operatorname{Coll}\left(\kappa^{+},<\kappa^{++}\right)$is equivalent to $\operatorname{Add}\left(\kappa^{+}, \kappa^{++}\right)$.

We factor $j: V \longrightarrow M$ through the ultrapower by the normal measure $U$ induced by $j$. Let $M_{0}=U l t(V, U)$. As usual we have an embedding $i: V \longrightarrow M_{0}$ and a map $k: M_{0} \longrightarrow M$ such that $k \circ i=j$. Since $\operatorname{crit}(k)>\kappa$ and $\kappa \in \operatorname{dom}(j(f))$, we see that $\kappa \in \operatorname{dom}(i(f))$.

Let $\mu=i(f)(\kappa)$. Consider the iteration $i\left(\mathbb{P}_{\kappa}\right)$ and let $\lambda$ be the least point greater than $\kappa$ in the support of this iteration. $\lambda$ is an $M_{0^{-}}$ inaccessible closure point of $i(f)$, so in particular $\lambda>i(f)(\kappa)=\mu$. We note also that at stage $\kappa$ the iteration $i\left(\mathbb{P}_{\kappa}\right)$ adds a generic for $\operatorname{Coll}\left(\kappa^{+},<\mu\right)$.

It is easy to see that $M_{0}[G]$ is closed under $\kappa$-sequences in $V[G]$. Working in $M_{0}[G]$, let $\mathbb{R}$ be the term forcing consisting of $\operatorname{Coll}\left(\kappa^{+},<\right.$ $\mu)$-names for elements of the factor forcing $i\left(\mathbb{P}_{\kappa}\right)_{\kappa+1, i(\kappa)}$. Standard arguments tell us that in $M_{0}[G]$ the poset $\mathbb{R}$ is $\lambda$-closed and has $i(\kappa)$ antichains. We may therefore build $H^{*} \in V[G]$ which is $\mathbb{R}$-generic over $M_{0}[G]$.

Since $M$ is the ultrapower of $V$ by a $\left(\kappa, \kappa^{++}\right)$-extender it follows that $M=\left\{j(f)(a): f \in V, \operatorname{dom}(f)=[\kappa]^{<\omega}, a \in\left[\kappa^{++}\right]^{<\omega}\right\}$. Since $j$ can be factored as $k$ composed with $i$, this implies that $M=\{k(F)(a): F \in$ $\left.M_{0}, a \in\left[\kappa^{++}\right]^{<\omega}\right\}$. Since $k(\mu)=j(f)(\kappa)=\kappa^{++}$we may consider the domain of the functions $F$ in this representation of $M$ to be $[\mu]^{<\omega}$. It is now routine to lift $k$ to a map $k^{+}: M_{0}[G] \longrightarrow M[G]$, and also to see that

$$
M[G]=\left\{k^{+}(F)(a): F \in M_{0}[G], a \in\left[\kappa^{++}\right]^{<\omega}, \operatorname{dom}(F)=[\mu]^{<\omega}\right\} .
$$

Since $\mathbb{R}$ is $\lambda$-closed in $M_{0}[G]$ we may transfer $H^{*}$ along $k^{+}$to get $H^{* *}$, which is generic over $M[G]$ for the term forcing consisting of $\operatorname{Coll}\left(\kappa^{+},<\kappa^{++}\right)$-terms for elements of $j\left(\mathbb{P}_{\kappa}\right)_{\kappa+1, j(\kappa)}$. We may now use the generic $g$ to realise $H^{* *}$, and obtain $H$ which is generic over $V[G * g]$ for $j\left(\mathbb{P}_{\kappa}\right)_{\kappa+1, j(\kappa)}$.

It is routine to lift $j$ to $j^{+}: V[G] \longrightarrow M[G * g * H]$, and to see that $M[G * g * H]=\left\{j^{+}(f)(a): f \in V[G * g], a \in\left[\kappa^{++}\right]^{<\omega}, \operatorname{dom}(f)=[\kappa]^{<\omega}\right\}$. 
Since $g$ is generic for $\kappa^{+}$-closed forcing we may transfer it along $j^{+}$ to get a generic $g^{+}$, and then lift again to get $j^{++}: V[G * g] \longrightarrow$ $M\left[G * g * H * g^{+}\right]$. It is easy to see that $j^{++}$is the ultrapower by a $\left(\kappa, \kappa^{++}\right)$-extender.

Since $V[G] \models{ }^{\kappa} O N \subseteq M[G]$ and $g$ is generic for $\kappa^{+}$-closed forcing, it is easy to see that $V[G * g] \models{ }^{\kappa} M\left[G * g * H * g^{+}\right] \subseteq M\left[G * g * H * g^{+}\right]$. We claim that $\kappa$ is not measurable in $M\left[G * g * H * g^{+}\right]$. By closure it will suffice to see that $\kappa$ is not measurable in $M[G * g]$, and since $\mathbb{P}_{\kappa}$ is $\kappa$-Knaster we know that $\kappa$ is not measurable in $M[G]$. The following easy lemma is therefore sufficient.

Lemma 4.1. Let $2^{\kappa}=\kappa^{+}$and let $\mathbb{S}$ be $\kappa^{+}$-closed. If $\kappa$ is not measurable in $V$, then $\kappa$ is not measurable in $V^{\mathbb{S}}$.

Proof. Suppose for a contradiction that $\kappa$ is measurable in $V^{\mathbb{S}}$, and let $\dot{U}$ name a measure. Enumerate the power set of $\kappa$ as $\left\langle X_{\alpha}: \alpha<\kappa^{+}\right\rangle$and build a decreasing sequence $\left\langle p_{\alpha}: \alpha<\kappa^{+}\right\rangle$where $p_{\alpha}$ decides whether $X_{\alpha} \in \dot{U}$. Let $U_{0}=\left\{X_{\alpha}: \exists \alpha<\kappa^{+} p_{\alpha} \Vdash X_{\alpha} \in \dot{U}\right\}$ and check that $U_{0}$ is a measure on $\kappa$.

Relabelling our models and embeddings as we did at the end of the last stage, we are in the following situation: $\mathrm{GCH}$ holds and there is $j: V \longrightarrow M$ such that $\operatorname{crit}(j)=\kappa,{ }^{\kappa} M \subseteq M, \kappa$ is not measurable in $M, j$ is the ultrapower by a $\left(\kappa, \kappa^{++}\right)$-extender and $\kappa^{++}=\kappa_{M}^{++}$.

Stage Four: We now do a Reverse Easton iteration $\mathbb{P}_{\kappa}$ of length $\kappa$, adding a non-reflecting stationary set of ordinals of cofinality $\omega$ to each $V$-measurable $\alpha<\kappa$. See [2] for a detailed discussion of this kind of iteration.

Let $G$ be $\mathbb{P}_{\kappa}$-generic. Since $\kappa$ is not measurable in $M$ the forcing $j\left(\mathbb{P}_{\kappa}\right)$ does not act at $\kappa$, and so in $M[G]$ the factor forcing $j\left(\mathbb{P}_{\kappa}\right)_{\kappa, j(\kappa)}$ is highly strategically closed. By the usual arguments $M[G]$ is closed under $\kappa$-sequences in $V[G]$, and we may build $H \in V[G]$ which is $j\left(\mathbb{P}_{\kappa}\right)_{\kappa, j(\kappa)}$-generic over $M[G]$.

We may then lift $j$ to get $j^{+}: V[G] \longrightarrow M[G * H]$. By arguments similar to those we have used in previous stages, $M[G * H]$ is closed under $\kappa$-sequences inside $V[G]$ and $\kappa$ is not measurable in $M[G * H]$. Also $j^{+}$is the ultrapower by a $\left(\kappa, \kappa^{++}\right)$-extender, all the models we have mentioned agree on the value of $\kappa^{++}$, and $\mathrm{GCH}$ holds in $V[G]$.

We claim that $\kappa$ is the least measurable cardinal in $V[G]$. We start by observing that all $V$-measurable cardinals below $\kappa$ are no longer measurable. Suppose that $\lambda<\kappa$ and $\lambda$ is measurable in $V[G]$ but not in $V$; then $\lambda$ is not in the support of $\mathbb{P}_{\kappa}$, so $\lambda$ is measurable in $V\left[G_{\lambda}\right]$. Now $\lambda$ must be Mahlo in $V$ and so $\mathbb{P}_{\lambda}$ is $\lambda$-Knaster, but this 
is impossible since $\lambda$-Knaster forcing cannot create measurability at $\lambda$. For more details, we refer the reader to Lemma 3 of [2].

Relabelling once again, we are now in the following situation: $\mathrm{GCH}$ holds and there is $j: V \longrightarrow M$ such that $\operatorname{crit}(j)=\kappa,{ }^{\kappa} M \subseteq M, \kappa$ is not measurable in $M$ and is the least measurable in $V, j$ is the ultrapower by a $\left(\kappa, \kappa^{++}\right)$-extender and $\kappa^{++}=\kappa_{M}^{++}$.

Stage Five: We will now force with a certain term forcing. Let $\mathbb{P}_{\kappa}$ be the Reverse Easton iteration in which $\alpha^{++}$Cohen subsets are added to each inaccessible less than $\kappa$. Let $\dot{\mathbb{Q}}$ be a term for $\operatorname{Add}\left(\kappa, \kappa^{++}\right)_{V^{\mathbb{P}_{\kappa}}}$ and $\dot{\mathbb{Q}}_{0}$ be a term for $A d d\left(\kappa, \kappa^{+}\right)_{V^{\mathbb{P}} \kappa}$. Let $\dot{\mathbb{R}}$ be a term for $A d d\left(\kappa^{+}, \kappa^{++}\right)_{V^{\mathbb{P}} \kappa^{*} \dot{\mathbb{Q}}_{0}}$. Finally we define $\mathbb{R}^{*}$ to be the term forcing consisting of $\mathbb{P}_{\kappa} * \dot{\mathbb{Q}}_{0}$-terms for elements of $\mathbb{R}$.

Using the arguments of [3] it is possible to show that $\mathbb{R}^{*}$ is equivalent in $V$ to $A d d\left(\kappa^{+}, \kappa^{++}\right)_{V}$. In particular $\mathbb{R}^{*}$ does not collapse cardinals or add any $\kappa$-sequences of ordinals. It follows that if in $V^{\mathbb{R}^{*}}$ we compute the Reverse Easton iteration to add $\alpha^{++}$subsets to each inaccessible $\alpha \leq \kappa$, we end up with a forcing which is isomorphic to $\mathbb{P}_{\kappa} * \dot{\mathbb{Q}}$. Let $G^{*}$ be $\mathbb{R}^{*}$-generic over $V$.

We now claim that forcing with $\mathbb{P}_{\kappa} * \dot{\mathbb{Q}}$ over $V\left[G^{*}\right]$ preserves the measurability of $\kappa$. To see this let $G * g$ be $\mathbb{P}_{\kappa} * \dot{\mathbb{Q}}$-generic over $V\left[G^{*}\right]$. We note that a fortiori $G * g$ is $\mathbb{P}_{\kappa} * \dot{\mathbb{Q}}$-generic over $V$. We set $g_{0}$ to be the restriction of $g$ to $\mathbb{Q}_{0}$, so that $G * g_{0}$ is $\mathbb{P}_{\kappa} * \dot{\mathbb{Q}}_{0}$-generic over $V$.

We use $G * g_{0}$ to realise the term generic $G^{*}$, thereby obtaining $h$ which is $\mathbb{R}$-generic over $V\left[G * g_{0}\right] . V[G * g]$ is a $\kappa^{+}$-c.c extension of $V\left[G * g_{0}\right]$ and $\mathbb{R}$ is $\kappa^{+}$-closed in $V\left[G * g_{0}\right]$, so by Easton's lemma $h$ is $\mathbb{R}$-generic over $V[G * g]$.

By Woodin's argument described in Section $3 \kappa$ is measurable in $V[G * g * h]$. By Easton's lemma and the fact that $\mathbb{P}_{\kappa} * \dot{\mathbb{Q}}$ is $\kappa^{+}$-c.c. we know that the power set of $\kappa$ in $V\left[G * g \times G^{*}\right]$ is equal to the power set of $\kappa$ in $V[G * g]$, which in turn is equal to the power set of $\kappa$ in $V[G * g * h]$. So $\kappa$ is measurable in $V\left[G * g \times G^{*}\right]$.

We can now prove the main result of this paper.

Theorem 4.2. If it is consistent that there exists a cardinal $\lambda$ of Mitchell order $\lambda^{++}$, then it is consistent that if $\kappa$ is the least measurable cardinal then $2^{\kappa}=\kappa^{+}$and $\kappa$ remains the least measurable cardinal after adding $\kappa^{++}$Cohen subsets of $\kappa$.

Proof. Consider the intermediate model $V\left[G * g_{0} \times G^{*}\right]$ from Stage Five. By Levinski's argument from Section $3 \kappa$ is measurable in this model, and familiar arguments give us that in this model $\kappa$ is the least measurable and $2^{\kappa}=\kappa^{+}$. 
It is also easy to see that $V\left[G * g_{0} \times G^{*}\right]$ has the same $<\kappa$-sequences of ordinals as $V[G]$, so that $A d d\left(\kappa, \kappa^{++}\right)_{V[G]}=A d d\left(\kappa, \kappa^{++}\right)_{V\left[G * g_{0} \times G^{*}\right]}$. Clearly $V\left[G * g \times G^{*}\right]$ can be viewed as an extension of $V\left[G * g_{0} \times G^{*}\right]$ by this poset.

We have shown that the model $V\left[G * g_{0} \times G^{*}\right]$ is as required.

\section{REFERENCES}

[1] A. Apter, Forcing the least measurable to violate GCH, Mathematical Logic Quarterly 45 (1999), 551-560.

[2] A. Apter and J. Cummings, Identity crises and strong compactness, Journal of Symbolic Logic 65 (2000), 1895-1910.

[3] J. Cummings, A model in which GCH holds at successors but fails at limits, Transactions of the American Mathematical Society 329 (1992), 1-39.

[4] M. Gitik, Changing cofinalities and the non-stationary ideal, Israel Journal of Mathematics 56 (1986), 280-314.

[5] M. Gitik, The negation of the singular cardinal hypothesis from $o(\kappa)=\kappa^{++}$, Annals of Pure and Applied Logic 43 (1989), 209-234.

[6] M. Gitik, A club of former regulars, Journal of Symbolic Logic 64 (1999), 1-12.

[7] J. Hamkins, The lottery preparation, Annals of Pure and Applied Logic 101 (2000), 103-146.

[8] J.-P. Levinski, Filters and large cardinals, Annals of Pure and Applied Logic 72 (1995), 177-212.

[9] W. Mitchell, Sets constructible from sequences of ultrafilters, Journal of Symbolic Logic 39 (1974), 57-66.

[10] W. Mitchell, Sets constructed from sequences of measures: revisited, Journal of Symbolic Logic 48 (1983), 600-609.

[11] W. Mitchell, The core model for sequences of measures I, Mathematical Proceedings of the Cambridge Philosophical Society 95 (1984), 229-260.

Department of Mathematics, Baruch College of CUNy, New York NY 10010

E-mail address: awabb@cunyvm.cuny.edu

URL: http://math.baruch.cuny.edu/ apter

Department of Mathematical Sciences, Carnegie Mellon UniverSity, Pittsburgh PA 15213

E-mail address: jcumming@andrew.cmu.edu

URL: http://www.math.cmu.edu/users/jcumming/ 